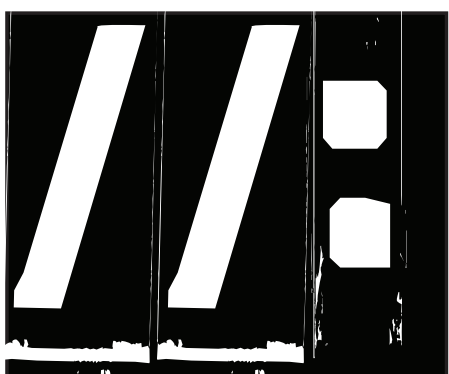

educa"ción y co"municación 10: 125-131 Mayo 2015

\section{LA COMUNICACIÓN EN LOS GRUPOS DE APOYO MUTUO [GAM]: UNA ESTRATEGIA INCLUSIVA DE APOYO}

\section{Communication in Mutual Support Team [GAM ]: an inclusive strategy to support}

Resumen:

Presentamos en este artículo una experiencia innovadora en el ámbito del Apoyo educativo: la creación, formación y desarrollo de Grupos de Apoyo Mutuo (GAM) en los colectivos educativos de profesores, familias y alumnado. Este proyecto se enmarca en una investigación $\mathrm{I}+\mathrm{D}+\mathrm{i}$ titulada: Escuelas que caminan hacia la inclusión educativa: trabajar con el apoyo educativo para promover el cambio, que tiene como meta la mejora de los procesos y estrategias de apoyo. Usando una metodología cualitativa caracterizada por ser descriptiva y participativa hemos podido conocer y analizar la dinámica de trabajo de las sesiones de apoyo que desarrollan los GAM. Señalamos finalmente algunas conclusiones vinculadas con los procesos de comunicación.

Palabras claves: Apoyo colaborativo, Educación Inclusiva, Mejora Escolar

\title{
Abstract:
}

This article presents an innovative experience in the field of Education support the creation, training and development Mutual Support Team (GAM) in educational groups of teachers, families and pupils. This project is part of an investigation $\mathrm{I}+\mathrm{D}+\mathrm{i}$ titled: Schools walking towards inclusive education: working with educational support to promote change, which aims to improve processes and support strategies. Using a qualitative methodology characterized by being descriptive and participatory we have been able to understand and analyze the dynamics of work support sessions developed by GAM. We note finally some conclusions related to communication processes. Keywords: Collaborative support, Inclusive Education, School Improvement

Recibido: 13/12/2014 Revisado: 21/1/2015 Aceptado: 22/2/2015 Publicado: 1/5/2015 


\section{II: Educación para la diversidad y comunicación}

\section{Introducción}

$\mathrm{L}^{2}$ a colaboración y la comunicación en el seno de las comunidades educativas, son sin duda dos focos de convergencia en el que teóricos y prácticos, investigadores, padres y profesores coincidimos al tratar de identificar las claves para avanzar en la búsqueda de la mejora escolar. Parece claro que el trabajo en grupo, la colaboración y la comunicación puede mejorar de forma sustantiva la respuesta que la escuela da a sus alumnos. Hasta tal punto es así que podríamos decir que crear comunidades inclusivas, de todos y para todos, es crear comunidades de comunicación y colaboración. Pero no es fácil desarrollar procesos comunicativos efectivos ni desarrollar un estilo de comunicación adecuado. Es necesario, como señalan distintos autores, adquirir competencias comunicativas verbales y no verbales (Sanz, 2005; Barrio y Borragán, 2011; Álvarez Núñez, 2012).

Por otra parte, actualmente constatamos que numerosos centros escolares han emprendido experiencias y proyectos de cambio intentando que la educación sea cada vez más una educación de equidad y de calidad para todos. Estas experiencias siendo bien distintas entre sí (hay diferencias en el tema concreto que abordan, el perfil profesional de los participantes, el nivel educativo, el impacto, etc.) han ido señalando algunas de las claves para el desarrollo de prácticas educativas y procesos de asesoramiento inclusivos, de tal modo que se han identificado una serie de rasgos o características que califican y cualifican a las escuelas inclusivas frente a las que podríamos llamar selectivas o excluyentes (Ainscow, 2001; Stainback y Stainback, 1999; Echeita y Ainscow, 2011; Echeita, Simón, López y Urbina, 2013). E igualmente en el terreno del asesoramiento y apoyo autores como Marcelo y López Yánez, (1997) señalaban en el inicio del movimiento de Escuelas inclusivas, el camino del cambio hacia unas escuelas "generadoras de aprendizaje" para todos. Desde esta perspectiva, los Grupos de Apoyo Mutuo (GAM) se constituyen como estructuras de apoyo de/y para colegas (profesores, familias y alumnos) para responder a las distintas necesidades de apoyo en cada colectivo (Gallego Vega, 2013)

Los antecedentes de esta experiencia lo encontramos en el contexto anglosajón como "Teacher Support Team” (Creese, Daniels, y Norwich, 1997) y en nuestro contexto como "Grupo de Apoyo entre Profesores" (Parrillla y Daniels, 1998; Gallego Vega, 2002) restringido sólo al colectivo de profesores. Su objetivo principal era el apoyo o ayuda que los profesores se prestaban entre sí para la resolución de problemas relacionados con la atención a la diversidad, y su meta secundaria era la creación de espacios y foros de conexión y colaboración entre profesores. Los resultados positivos de estas experiencias motivaron que el proyecto que aquí presentamos se hiciera extensivo en cuanto a participantes (la incorporación de familia y alumnos) y contenido de la ayuda (nos sólo para problemas vinculados con la atención a la diversidad sino para cualquier problema educativo).

\section{Los centros participantes}

En esta experiencia participan 6 centros andaluces de las Provincias de Málaga, Cádiz y Sevilla de distintas etapas educativas: 3 centros de Secundaria, 2 de Infantil-Primaria y uno de Educación Infantil exclusivamente. Cada centro se ha "apropiado" del proyecto y lo ha hecho suyo en el sentido de formar los GAM 


\section{1/: La comunicación en los Grupos de Apoyo Mutuo - (GAM): una estrategia inclusiva de apoyo}

que han creído convenientes según sus necesidades; y la composición de los miembros que lo forman. La flexibilidad del proyecto permite que los propios participantes lo adecuen a sus necesidades y características propias: en total son 6 GAM de profesores, 2 GAM de familias y 2 GAM de alumnos.

\section{Los Grupos de Apoyo Mutuo}

Los Grupo de Apoyo Mutuo son unas estructuras y sistemas de apoyo colaborativo, formado y gestionado por personas de un mismo colectivo y centro, que ayudan a otros colegas que voluntariamente acuden al grupo en el análisis y búsqueda de soluciones a los problemas que se les planteen. Algunas de las señas de identidad de este modelo de apoyo son:

- Parten de la idea de que el conocimiento y experiencia del profesorado, de las familias y de los estudiantes son válido para resolver autónomamente muchos de los problemas que se dan en los centros educativos.

- Proporcionan una oportunidad para que los profesores, familias y alumnos de un mismo centro compartan, analicen y resuelvan conjuntamente sus problemas, sin necesidad de recurrir sistemáticamente a las ayudas o asesoramiento a expertos del apoyo interno y externo al centro.

- Introduce la idea de un modelo de apoyo inclusivo basado en el desarrollo y apoyo que proporcionan las redes naturales de apoyo.

- Una vía para iniciar y desarrollar un enfoque colaborativo y de comunicación en los centros educativos.

El desarrollo de estos grupos en la práctica está demostrando que son útiles y válidos para afrontar cual- quier situación problemática educativa y no educativa que se presente.

\section{Miembros de los Grupos de Apoyo Mutuo}

A nivel teórico, cada GAM debe de estar formado por tres miembros ya sea de profesor/a, madre/padre o familiar y alumno o alumnos. El rol que cada uno adopta en las sesiones de apoyo es distinto: uno asume el rol de secretario (recoge la documentación del caso en una hoja de recogida de datos), otro toma el rol de coordinador de la sesión y el otro apoya al resto. Aunque ésta es la composición recomendada teóricamente, en su desarrollo la diversidad de los grupos de apoyo (en cuanto a número de miembros y roles desempeñados) es significativa, desde grupos formados por más de cuatro personas, hasta grupos cuyos miembros ostentan algún cargo administrativo en el centro. La teoría también señala la pertinencia de que los miembros del grupo roten y cambien cada año para que no se conviertan en "Grupos de Expertos" y permita de forma democrática que cualquier profesor, familia o alumno pueda pertenecer al grupo. Esto permite promover el dinamismo y evita la identificación de las tareas de apoyo con personas concretas dentro del centro lo que ayuda a adquirir una visión y sentido amplio de apoyo.

\section{La dinámica y comunicación en los Grupos de Apoyo Mutuo}

El proceso típico de trabajo de un GAM (Gallego, 2002), se inicia una vez que un/varios miembros de la comunidad educativa manifiesta/n su interés en acudir a discutir algún problema con el Grupo de Apoyo Mu- 


\section{II: Educación para la diversidad y comunicación}

tuo, (normalmente se pone en contacto informalmente con algún miembro del grupo para concertar el día y hora de reunión). En el día establecido de reunión, se reúnen los miembros del grupo con la persona que demanda ayuda, quien automáticamente pasa a ser considerado como un miembro más del grupo (a efectos del análisis de su problema). Utilizando el diálogo y la comunicación en el seno del grupo, colaborativamente se analizan las distintas vertientes y perspectivas del problema que el demandante plantea, con el fin de buscar estrategias que se puedan poner en práctica para la posible solución de la situación planteada. Al ser un grupo que trabaja desde los presupuestos de la colaboración y desde una orientación ecológica ante el análisis y comprensión de problemas, las estrategias que adoptan deben ser coherentes y sensibles con esos planteamientos y adecuadas a las limitaciones de tiempo, espacio y recursos que posean los centros. Así el esquema típico de trabajo que utilizan los Grupos de Apoyo Mutuo es el llamado modelo de acción para el análisis y resolución colaborativa de Problemas (figura 1).

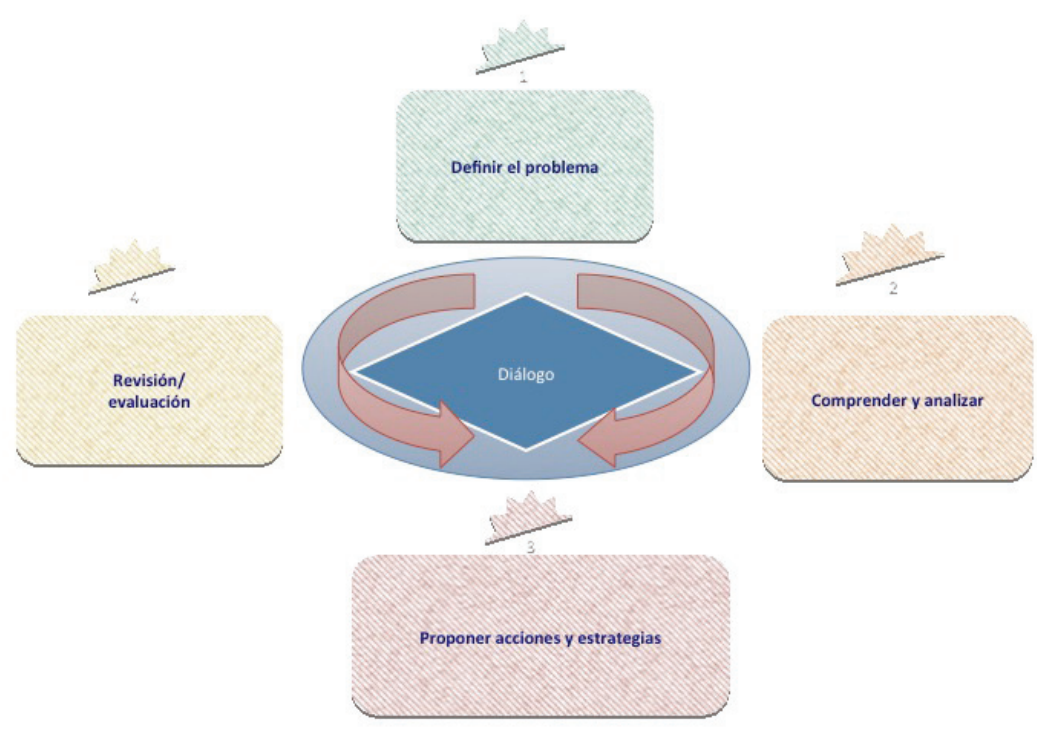

Figura 1. Modelo de resolución de problemas (Adaptación de Creese, Daniels y Norwich, 1997) 


\section{1/: La comunicación en los Grupos de Apoyo Mutuo 1.- (GAM): una estrategia inclusiva de apoyo}

El desarrollo de cada una de las fases del modelo conlleva un proceso de toma de decisiones en el que los miembros del grupo, junto con el demandante que hace la consulta, participan en un diálogo basado en la confianza y además se establece el compromiso de que lo que se trata en las reuniones, es confidencial. Las actividades y estrategias que se ponen en marcha en cada una de estas fases o pasos son:

\section{Definir el problema.}

Cuando una o varias personas (pueden presentar la demanda más de una persona, según el caso) acude a un GAM su demanda suele ser real (no es ni forzada ni burocrática como suele ocurrir en otros modelos de apoyo), parte del deseo de afrontar un problema en el que está implicado y del compromiso de participar en la puesta en práctica de las estrategias que se acuerden. El hecho de que el grupo al que acude el demandante lo compongan colegas (que comparten una misma cultura institucional y que quizás conozcan hasta el problema como aparece en la siguiente cita), y la voluntariedad del proceso garantiza que la relación que se establezca en el grupo y el diálogo que desarrollen se oriente realmente a un trabajo práctico sobre el problema planteado.

"Entrev.- porque el grupo de apoyo tenía más o menos conocimiento del problema que tú planteabas... Prof. 2.- ¡Hombre! Más o menos sí, porque tú ten en cuenta que en este colegio como estamos tan poca gente, pues a los críos se les conoce..., yo conozco prácticamente a todos los niños del colegio, y sabes más o menos cómo es uno, cómo es otro, pues igual les pasa a los otros compañeros, ¿sabes?” (Entrev. Prof. Demandante)

El demandante que realiza la demanda al Grupo de Apoyo, puede tener identificado y definido claramen- te el problema o irse definiendo a medida que lo describe al grupo. A veces sólo con definir el problema pueden surgir las estrategias para su solución. Así pues, cuando se habla de identificar el problema nos referimos a centrarlo y aclararlo, llegando a definirlo en términos que faciliten el paso a un análisis más en profundidad del mismo.

\section{Comprender y analizar el problema.}

Esta fase está estrechamente unida a la de identificación del problema, y aunque a nivel teórico las desarrollemos por separado, la práctica de los Grupos de Apoyo Mutuo nos muestra que muy a menudo, se dan conjuntamente: a medida que se describe el problema se va realizando el análisis del mismo.

Es importante en esta fase, que los componentes del grupo se apropien y comprendan el problema, señalando las dimensiones o contextos que pueden identificarse en el mismo. En el diálogo compartido que se establece con el demandante, los miembros del Grupo de Apoyo Mutuo pueden utilizar preguntas que aclaren y definan la situación.

"en la conversación que establecemos para aclarar y ampliar esta situación que nos presenta y así poder plantearnos respuestas a sus pregunta, recabamos la siguiente información: en lo que va de curso ya hemos abierto dos expedientes a alumnos con comportamiento graves de conducta (...), (etc)" (Prof. Miembro del GAM. Autoinforme de un caso)

\section{Proponer acciones y estrategias.}

Una vez realizado el análisis del problema, se entra en la siguiente fase, consistente en planificar las acciones o estrategias que se desarrollaran para afrontar el mismo. Es conveniente partir del análisis del problema realizado, éste servirá de guía para planificar los ám- 


\section{II: Educación para la diversidad y comunicación}

bitos de intervención que se van a desarrollar. En esta fase el Grupo de Apoyo tiene que tomar decisiones tanto en cuanto a las alternativas o estrategias que se adopten como a la priorización de las mismas.

El proceso a seguir en esta parte es un proceso lineal: en primer lugar detectar las dimensiones o ámbito de intervención, a continuación priorizar las mismas, y elaborar un plan de acción, diseñando estrategias y alternativas que sean viables para la resolución del problema, planificando los recursos necesarios para su puesta en marcha, previendo el tiempo y espacio de realización de las mismas, etc.

"pues acordamos, por ejemplo una idea: pues en el recreo vamos a quitarle los partidos y vamos a estar pendientes para que no haya disputas para que no estén diciendo palabrotas y vamos a intentar cortar, pues eso para que les diera tiempo a beber, a relajarse, pues eso se acordó antes de terminar la sesión, se llevó a acabo, entonces pues depende de lo que fuese pues se aportaban ideas o se llegaba ya a conclusiones" (Entrev. Prof. Demandante)

\section{Revisión y evaluación de los resultados}

En las sesiones de revisión o seguimiento del caso, se evalúa el proceso seguido en la puesta en marcha de las estrategias acordadas en el Grupo de Apoyo Mutuo. Se revisan tanto el proceso como los resultados que se hayan obtenido, y las dificultades e incidencias que pudieran haber surgido en el desarrollo de las mismas. En base a esta valoración se pueden adoptar nuevas estrategias, utilizar las mismas con las adaptaciones que se crean convenientes o dar por cerrado el caso si el problema se ha resuelto o lleva un buen camino de resolución.

Toda esta dinámica de trabajo se refleja en una hoja de registro, diseñada por el propio grupo, que le sirve de control y evaluación del trabajo desarrollado por el grupo. El clima que se mantiene en estas reuniones se caracteriza por ser colaborativo, evitando en todo momento la típica relación de desigualdad, basada en relaciones jerárquicas y expertas entre los miembros del grupo y el demandante. Si algo caracteriza a los GAM, es el reconocimiento de la igualdad entre sus miembros y el respeto a las distintas perspectivas y puntos de vistas que cada uno puede aportar al caso que es la base sobre la que se asienta el trabajo colaborativo.

"y lo bueno es que a mí lo que me ha gustado es que no están ellos ahí y nosotros aquí, sino que es una mesa camilla, me entiendes?... Ellos aprenden también de nosotros, sobre todo el día ese que nos reunimos todos fue genial" (Entrev. Prof. Demandante).

\section{A modo de conclusión}

Centrándonos en la dinámica y comunicación que se desarrolla en el seno de los GAM y sin entrar en un análisis exhaustivo de las distintas aportaciones que los miembros de los Grupos de Apoyo Mutuo y otros participantes en el Proyecto atribuyen, sí quisiéramos ejemplificar algunas de las aportaciones más destacadas.

En relación con la adquisición y desarrollo de habilidades sociales, los miembros de los grupos de apoyo declaran que son más tolerantes ante opiniones opuestas o divergentes, practican y aprenden a ponerse en el lugar del otro (empatía), respetan el turno de palabra y reaprenden a "guardar silencio". En relación con el desarrollo de competencias comunicativas, adquieren destrezas en "la escucha activa", son más conscientes de la emisión de mensajes obstructores/facilitadores 


\section{1/: La comunicación en los Grupos de Apoyo Mutuo 11. (GAM): una estrategia inclusiva de apoyo}

\section{Divulgatio}

de la comunicación así como del lenguaje gestual y corporal que pueden dificultar o favorecer la comunicación. Por último, a nivel personal declaran que se sienten mejor (mayor autoestima), reconocen que es mejor resolver problemas en grupo que "en solitario" (se incrementa la pertenencia y cohesión grupal) y se sienten ayudados y comprendidos por sus colegas (lo que minimiza los niveles de estrés que genera un problema).

\section{Referencias}

Ainscow (2001). Desarrollo de escuelas inclusivas. Madrid: Narcea.

Creese, A., Daniels, H. y Norwich, B. (1997). Teacher support team in secondary schools. DfEE Report.

Echeita, G. y Ainscow, M. (2011). "La educación inclusiva como derecho. Marco de referencia y pautas de acción para el desarrollo de una revolución pendiente". En Tejuelo, 12; 26-46.
Echeita, G.; Simón, S.; López, M. y Urbina, C. (2013). "Educación inclusiva. Sistemas de Referencia, coordenadas y vórtices de un proceso dilemático". En: M.A.Verdugo y R.L. Shalock (Coords.). Discapacidad e Inclusión. Salamanca, Amarú; 329-357.

Marcelo, C. y López Yánez, J. (Coords.) (1997). Asesoramiento curricular y organizativo en educación. Barcelona: Ariel.

Gallego, C. (2002). "El apoyo entre profesores como actividad educativa inclusiva." En Revista de Educación, 327; 83-105.

Gallego Vega, C. (2013). "Una formación compartida entre familias y profesores para el desarrollo de Grupos de Apoyo Mutuo". En Revista de Investigación en educación. 11(3); 109-119.

Parrillla, A. y Daniels, H. (Coords.) (1998). Creación $y$ desarrollo de grupos de apoyo entre profesores. Bilbao: Mensajero.

Stainback, S. y Stainback, W. (1999). Aulas Inclusivas. Madrid: Narcea. 